Published in final edited form as:

Curr Opin Cardiol. 2015 May ; 30(3): 250-258. doi:10.1097/HCO.0000000000000163.

\title{
Advances in the pathophysiology and treatment of heart failure with preserved ejection fraction
}

\author{
Sara Tannenbaum and Gabriel T. Sayer \\ Section of Cardiology, University of Chicago, Chicago, Illinois, USA
}

\begin{abstract}
Purpose of review-With the failure of multiple trials to identify a successful therapy for heart failure with preserved ejection fraction (HFpEF), attention has shifted to defining specific phenotypes within the HFpEF spectrum in an effort to develop a targeted approach to treatment. Here we summarize the most recent studies investigating the pathophysiology and clinical features of $\mathrm{HFpEF}$, and discuss recent clinical trials in the context of developing treatments that look toward the underlying cause of this disorder.
\end{abstract}

Recent findings-Advances in basic science and clinical research have further characterized HFpEF, identifying multiple pathophysiological mechanisms that ultimately lead to exercise intolerance and volume overload. The success of small studies focused on specific subsets of the HFpEF population has promoted the concept that there may not be one treatment strategy that can universally be applied to HFpEF.

Summary-HFpEF is associated with significant morbidity and mortality and accounts for approximately half of patients with chronic heart failure. HFpEF is a complex disease, encompassing a diverse cohort of patients and marked by the presence of multiple etiological mechanisms. The failure to develop successful therapies for the management of HFpEF may be because of inadequate standardization of the HFpEF diagnosis, overly broad inclusion criteria and inadequate differentiation of disease subtypes. Given the heterogeneity among patients with HFpEF, much of the current research is focused on understanding of pathophysiology and identifying disease phenotypes that may respond to a targeted treatment approach. Several newer approaches, including neprilysin inhibition and device therapy, offer promise for a new era of HFpEF treatment.

\section{Keywords}

diastolic dysfunction; heart failure with preserved ejection fraction; renin-angiotensin-aldosterone blockade; targeted therapy

Correspondence to Gabriel T. Sayer, Section of Cardiology, University of Chicago, 5841 S. Maryland Avenue MC 6080, Chicago IL 60637, USA. Tel: +1 773702 9396; gsayer@medicine.bsd.uchicago.edu.

Conflicts of interest

There are no conflicts of interest.

Financial support and sponsorship

None. 


\section{INTRODUCTION}

Among patients with chronic heart failure, approximately 50\% have a preserved ejection fraction (HFpEF) [1]. The prevalence of heart failure with a preserved ejection fraction (HFpEF) is expected to increase with more clinical recognition of this disease and the aging of the population [2]. HFpEF has a substantial impact on health care costs, and patients with HFpEF have reduced survival, although overall mortality is not as severe as for patients with an overt systolic dysfunction [3"]. No clinical trial to date has identified a therapy that improves survival in HFpEF. This may be because of the complex pathophysiology of $\mathrm{HFpEF}$ as well as failures of trial design. Unlike patients with HFrEF, whose symptoms can be directly tied to the consequences of systolic dysfunction, the cause of dyspnea in patients with HFpEF has remained more opaque. HFpEF is a heterogeneous disorder with multiple identified mechanisms and a wide variety of clinical presentations. Identifying a narrow set of disease-specific inclusion criteria for clinical studies has been a challenge. In addition, HFpEF patients have a high prevalence of comorbidities (hypertension, diabetes, chronic kidney disease, obesity) that can contribute significantly to the morbidity and mortality associated with the disease, making the identification of HFpEF-specific therapies more difficult. Much of the current research in this field is focused on the underlying pathophysiological mechanisms, with the goal of identifying disease phenotypes that may respond to a targeted treatment approach. In this review, we summarize the most recent data on the pathophysiology of HFpEF and discuss emerging evidence from clinical trials of HFpEF therapies.

\section{KEY POINTS}

- $\quad \mathrm{HFpEF}$ represents approximately half of all heart failure, and leads to significant morbidity and mortality.

- $\quad$ Patients with HFpEF typically have multiple comorbidities, and disease presentation varies depending on the comorbidity profile.

- $\quad$ Exercise intolerance is the primary manifestation of HFpEF and has multiple etiological mechanisms, including diastolic dysfunction, chronotropic incompetence, pulmonary hypertension and subtle abnormalities of systolic dysfunction.

- Unlike HFrEF, no clinical trial to date has identified a therapy that improves survival in HFpEF. This may be related to inadequate understanding of pathophysiology and the application of broad therapies to a heterogeneous disorder.

- $\quad$ Classification of HFpEF phenotypes will allow the use of targeted therapies such as exercise training or rate-adaptive pacing for subgroups of the HFpEF population. 


\section{PATHOPHYSIOLOGY AND CLINICAL FEATURES OF HEART FAILURE WITH PRESERVED EJECTION FRACTION}

$\mathrm{HFpEF}$ is characterized by abnormalities of both diastolic and systolic function that result in exercise intolerance. Animal models have highlighted the role of the cytoskeletal protein titin in promoting passive stiffness that underlies the development of diastolic dysfunction [4",5',6]. Diastolic dysfunction is considered a crucial component of HFpEF, and impaired ventricular relaxation has been well documented by both echocardiography and invasive hemodynamics [7,8]. Recently, human studies have focused on measuring abnormalities of cardiac performance not captured by ejection fraction, a highly load-dependent measure of systolic function. Using speckle tracking imaging, these studies have demonstrated that $\mathrm{HFpEF}$ is associated with abnormalities of global and longitudinal strain, dyssynchrony and impaired left atrial function $\left[9^{\mathbf{*}}, 10^{\mathbf{*}}, 11^{\mathbf{*}}\right]$. In addition, a series of elegant exercise studies have demonstrated marked impairment of chronotropy and cardiovascular reserve among HFpEF patients $[12,13]$.

The comorbidities associated with HFpEF play a significant role in its morbidity and mortality $\left[14^{\boldsymbol{}}, 15\right]$. Both atrial fibrillation and renal dysfunction are associated with worsened parameters of systolic and diastolic function, and the presence of atrial fibrillation has a significant impact on exercise capacity [16",17"]. Diabetic patients have an increased burden of comorbidities, higher levels of inflammatory markers, worsened functional status and increased risk of hospitalizations [18 $]$. Likewise, HFpEF patients with coronary artery disease have an increased risk of mortality and subsequent decline in systolic function [19"]. Noninvasive measures of endothelial function have also been associated with adverse events in the HFpEF population [20]. Paulus and Tschope [21"'] have proposed a unifying hypothesis that comorbidities underlie the syndrome of HFpEF by inducing a proinflammatory state with multiple consequences, including endothelial dysfunction, dysregulation of myocyte hypertrophy and collagen deposition.

Designing therapeutic interventions for HFpEF has been complicated by the heterogeneity of this disorder. As a result, attention has been devoted to better defining HFpEF phenotypes. An autopsy study found that $19 \%$ of HFpEF subjects showed evidence of wildtype transthyretin amyloid deposition, which was associated with excess fibrosis [22"]. Identifying this subset of HFpEF will take on increased importance with the emergence of therapies that stabilize the transthyretin protein. One medication, tafamidis, has shown promise in the treatment of amyloid neuropathy and is currently under investigation in a Phase 3 trial for patients with familial or wild-type amyloid cardiomyopathy (NCT01994889). Large clinical trials with echocardiographic core laboratories have characterized the diversity of ventricular structure present in HFpEF, with most patients exhibiting concentric remodeling or concentric hypertrophy, but a significant percentage with eccentric hypertrophy [23"]. Sex differences are present, with women exhibiting increased left ventricular wall thickness and ventricular stiffness [24]. Increasing attention is being directed at the role of the right ventricle. Right ventricular dysfunction is a feature of HFpEF that carries a strong association with mortality, andmay be a good target for pulmonary vasodilator therapies $\left[25,26^{\mathbf{*}}-28^{\mathbf{*}}\right]$. 


\section{THERAPEUTIC TARGETS FOR HEART FAILURE WITH PRESERVED EJECTION FRACTION}

Clinical trials of therapies for HFpEF have explored multiple pathways, based on proposed pathophysiological mechanisms as well as extrapolation from treatments that have shown benefit in HFrEF. To date, no therapy has proven to improve survival in HFpEF. The mainstays of treatment are diuretics and guideline-directed management of comorbidities. The importance of extracellular volume management was highlighted by a subgroup analysis from the CardioMEMS Heart Sensor Allows Monitoring of Pressure to Improve Outcomes in NYHA Class III Heart Failure Patients (CHAMPION) trial, in which HFpEF patients with an implantable pulmonary artery pressure monitoring system had a 50\% reduction in the incidence of heart failure hospitalization over 18 months, primarily because of augmentation of diuretic therapy [29"- $]$. Exercise training has been studied as nonpharmacological therapy, and has shown success in improving exercise capacity in patients with exertional dyspnea, although it has failed to alter other pathophysiological abnormalities characteristic of $\mathrm{HFpEF}$ [30",31"].

\section{Targeting the renin-angiotensin-aldosterone system}

The renin-angiotensin-aldosterone system (RAAS) was the most obvious target for potential HFpEF therapies because of the experience with inhibition of RAAS in HFrEF as well as the association of neuro-hormonal activation with hypertension and volume retention. Preventing the adverse effects of angiotensin II [with angiotensin-converting enzyme (ACE) inhibitors or aldosterone receptor blockers (ARB)] was studied in three trials: Candesartan in Heart failure: Assessment of Reduction in Mortality and morbidity (CHARM)-Preserved, Perindopril in elderly people with chronic heart failure (PEP-CHF) and Irbesartan in Heart Failure with Preserved Ejection Fraction (I-Preserve) [32-34]. All three studies failed to find any mortality benefit from RAAS inhibition (Table 1). CHARMPreserved identified a reduction in heart failure hospitalizations, a secondary endpoint, with candesartan as compared with placebo, although the use of a left ventricular ejection fraction (LVEF) of $40 \%$ as the lower limit for the inclusion criteria raises the question of whether it was truly a HFpEF population that was studied. PEP-CHF found a reduction in multiple secondary endpoints, including exercise capacity and heart failure hospitalizations, with the ACE-inhibitor perindopril as compared with placebo [33]. However, PEP-CHF was a relatively small trial that was underpowered because of a low event rate, making it difficult to draw significant conclusions from secondary endpoints. The I-Preserve trial, which used an LVEF more than $45 \%$ as an inclusion criterion (and therefore may more closely represent a true HFpEF population), failed to find a difference in any of its secondary endpoints, including quality of life and heart failure hospitalizations [34].

Blocking the downstream effects of aldosterone is appealing in the treatment of $\mathrm{HFpEF}$ because of aldosterone's association with volume retention and ventricular fibrosis. Two trials have investigated aldosterone antagonism in HFpEF: Aldosterone Receptor Blockade in Diastolic Heart Failure (ALDO-DHF) and Treatment of Preserved Cardiac Function Heart Failure With an Aldosterone Antagonist (TOPCAT) (Table 1) [35,36"']. ALDO-DHF failed to find an improvement in functional outcomes, despite improvement in diastolic function 
(E/E') on echocardiography, and was not designed to look at hospitalizations or survival [35].

The TOPCAT trial is a large, randomized study that was powered to investigate the effect of aldosterone antagonism on clinical outcomes in HFpEF [36"']. In TOPCAT, patients with symptomatic heart failure, an LVEF of $45 \%$ or greater, and either a prior hospitalization for heart failure within the past year or an elevated natriuretic peptide level were randomized to spironolactone or placebo. A total of 3445 patients were randomized, the median age was 68 , and $50 \%$ were women. At a mean follow-up of 39 months, there was no significant difference in the primary endpoint of death from cardiovascular causes, aborted cardiac arrest or hospitalization for heart failure ( 18.6 versus $20 \%, P=0.14$ ). Although the primary endpoint was neutral, there was a lower incidence of hospitalization for heart failure in the spironolactone group (12.0 versus $14.2 \%, P=0.04)$.

TOPCAT's patients were reflective of a contemporary HFpEF population, with high rates of hypertension (91\%), obesity (55\%), diabetes (32\%), chronic kidney disease (38\%), atrial fibrillation $(35 \%)$ and coronary artery disease $(59 \%)$. The study may have been limited by having broad inclusion criteria and by the enrollment of patients at varying stages of disease progression, as there was marked geographical variation in event rates [37 ${ }^{\mathbf{*}}$ ]. A similar finding has recently been reported from a review of multiple $\mathrm{HFpEF}$ trials, suggesting discrepancies in the definition of HFpEF across study sites [38 ${ }^{\mathbf{}}$.

\section{Targeting pulmonary hypertension}

With the negative results of spironolactone in TOPCAT added to the previous failure of other RAAS inhibitors to alter mortality in HFpEF, the focus of clinical efforts has turned toward therapies that more specifically target pathophysiological abnormalities. Pulmonary hypertension has been described in a significant percentage of the HFpEF population, and is associated with increased morbidity and mortality, making pulmonary vasodilation an attractive therapeutic strategy [39"]. In addition to vasodilation, inhibition of phosphodiesterase- 5 has been shown to reverse cardiac hypertrophy, fibrosis and contractile dysfunction [40]. The use of sildenafil in HFpEF was initially examined in a small study by Guazzi et al., which randomized 44 patients with HFpEF and pulmonary hypertension to sildenafil or placebo [41]. Large improvements were seen in right atrial pressure, pulmonary artery pressure and right ventricular function after 6 months. Of note, baseline right atrial pressure was markedly elevated in this study $(23 \mathrm{mmHg})$, suggesting that this study population had severe right ventricular dysfunction and may not be reflective of the larger population with HFpEF.

The Phosphodiesterase-5 Inhibition to Improve Clinical Status and Exercise Capacity in Heart Failure with Preserved Ejection Fraction (RELAX) trial compared sildenafil with placebo in 216 patients with symptomatic heart failure, LVEF greater than 50\%, decreased peak oxygen consumption and either an elevated NT-proBNP or evidence of resting or exercise-induced elevation of filling pressures [42-" $]$. At a mean follow-up of 24 weeks, there was no difference between the two groups in the primary endpoint of change in peak oxygen consumption $(-0.20$ versus $-0.20 \mathrm{ml} / \mathrm{kg} / \mathrm{min}, P=0.90)$. There was also no difference in any of the secondary endpoints, including parameters of diastolic function, change in 6- 
min walk distance or clinical status as measured by a hierarchical composite score.

Pulmonary hypertension was not required for entry into the study, which may have limited the efficacy of a therapeutic strategy that targets the pulmonary vasculature. An ongoing study will assess the impact of sildenafil on pulmonary pressures in HFpEF patients with pulmonary hypertension documented by invasive hemodynamics (Table 2).

\section{Targeting heart rate}

Heart rate is a marker of sympathetic activation and has been correlated with adverse outcomes in HFpEF. A substudy of the I-Preserve trial found an inverse association between heart rate (in sinus rhythm) and the incidence of cardiovascular death or heart failure hospitalizations [44"]. Ivabradine, an $\mathrm{I}_{\mathrm{f}}$-channel inhibitor, works directly on the sinus node to reduce heart rate without negative inotropic effects. This approach holds promise in diastolic dysfunction, as slower heart rates permit greater time for ventricular filling, and may be particularly well suited for patients whose symptoms predominantly occur with exercise. Favorable data from an animal model indicated improvements in vascular stiffness, ventricular elastance, and diastolic function following selective heart rate reduction with ivabradine [ $\left.47^{\boldsymbol{H}}\right]$. One clinical study has recently reported on the short-term use of ivabradine in HFpEF [43"']. Sixty-one patients were randomized to take ivabradine or placebo for 7 days. Ivabradine therapy resulted in an increase in peak $\mathrm{VO}_{2}$ of $3.0 \pm 3.6 \mathrm{ml} / \mathrm{kg} / \mathrm{min}$ as compared with an increase of $0.4 \pm 1.2 \mathrm{ml} / \mathrm{kg} / \mathrm{min}$ for placebo $(P=0.003)$. Additionally, patients in the ivabradine arm had a reduction in the change of $\mathrm{E} / \mathrm{E}^{\prime}$ with exercise $(3.1 \pm 2.7$ prior to treatment as compared with $1.3 \pm 2.0$ following treatment; $P=0.004$ ), whereas there was no significant change in this parameter during exercise in the placebo group (Table 2).

\section{Targeting the natriuretic peptide system}

The natriuretic peptides play a crucial role in fluid homeostasis. They are released in response to ventricular stretch, resulting in vasodilation, natriuresis and myocardial relaxation. One method of augmenting natriuretic peptide activity is through the inhibition of neprilysin, a protease that degrades biologically active natriuretic peptides.

The Prospective Comparison of ARNI With ARB on Management of Heart Failure with Preserved Ejection Fraction (PARAMOUNT) trial was a Phase II trial that compared LCZ696 (an angiotensin receptor neprilysin inhibitor) with valsartan in 308 patients with symptomatic heart failure, LVEF of at least 45\% and elevated NT-proBNP levels [45]. At 12 weeks, NT-proBNP levels were significantly reduced in the LCZ696 arm compared with the valsartan group (ratio of change LCZ696/valsartan 0.77, $P=0.005$ ). At 36 weeks, NTproBNP levels remained reduced from baseline in the LCZ696 group; however, the difference between the study groups was no longer significant $(P=0.20)$. Heart failure symptoms were improved in a greater percentage of the LCZ696 group at 36 weeks $(P=0.05)$. Blood pressure was reduced to a greater extent with LCZ696, although a subsequent analysis suggested that the benefits observed in the trial were independent of the blood pressure effect [48]. Prospective Comparison of ARN with ARB Global Outcomes in Heart Failure with Preserved Ejection Fraction (PARAGON-HF), a large Phase III trial investigating the impact of LCZ696 on cardiovascular death and heart failure hospitalizations in HFpEF, is currently enrolling patients (Table 2). 


\section{FUTURE DIRECTIONS}

Table 2 outlines a selection of ongoing investigations of novel therapies for HFpEF. Renal denervation is an approach that has attracted significant attention. The potential benefits of renal denervation in the HFpEF population include suppression of the excess sympathetic activity associated with heart failure and stricter control of hypertension. Several studies are currently evaluating the effect of renal denervation on changes in ventricular structure and measures of diastolic function.

Soluble guanylate cyclase stimulators enhance production of cyclic guanosine monophosphate, inducing vasodilation and inhibiting the development of fibrosis. Riociguat was recently studied in pulmonary hypertension associated with HFrEF and failed to meet its primary endpoint of change in mean pulmonary artery pressure, although it did improve cardiac index and reduce pulmonary vascular resistance [49]. Acute Hemodynamic Effects of Riociguat in Patients with Pulmonary Hypertension Associated with Diastolic Heart Failure (DILATE-1), a small study of short-term administration of riociguat in HFpEF, failed to demonstrate a reduction in pulmonary artery pressures as compared with placebo, but did find an improvement in stroke volume and right ventricular end-diastolic area [27']. A Phase II study of vericiguat, Soluble Guanylate Cyclase Stimulator in Heart Failure Study (SOCRATES-PRESERVED), is in progress [46].

Device therapy for the treatment of HFpEF is an area of active investigation. Chronotropic incompetence has increased prevalence in the HFpEF population and limits the ability of HFpEF patients to augment their cardiac output during exercise [50]. Restoration of chronotropic competence has promise for reducing exercise-induced dyspnea in HFpEF, but unfortunately, a prospective assessment of rate-adaptive pacing was terminated because of insufficient enrollment [51]. Another approach is to pace the left atrium through a coronary sinus lead to restore left atrial filling in patients with 'atrial dyssynchrony syndrome,' which is characterized by interatrial conduction delay and increased left atrial stiffness. A pilot study showed increased 6-min walk distance during active pacing, as well as improvement in left atrial and left ventricular filling [52]. The Left Atrial Pacing in Diastolic Heart Failure trial will attempt to confirm these results in a larger cohort. Mechanical circulatory support has not been tested in HFpEF; however, a small series of left ventricular assist device therapy in patients with restrictive or hypertrophic cardiomyopathies suggested an increased incidence of right ventricular failure after implantation [53].

There are several structural devices in development for the treatment for HFpEF. Percutaneous creation of an interatrial shunt has been developed as a method to reduce left atrial pressure, with the aim of reducing exertional dyspnea. In a computer model, the addition of the interatrial shunt resulted in a reduction of pulmonary capillary wedge pressure by $3 \mathrm{mmHg}$ at rest and $11 \mathrm{mmHg}$ during peak exercise. These changes were accompanied by a decrease in left ventricular cardiac output and an increase in right ventricular cardiac output [54]. The InterAtrial Shunt Device (DC Devices, Inc., Tewksbury, MA, USA) is a percutaneously implanted 8-mm shunt and has been tested in a pilot study of 11 patients with an LVEF higher than $45 \%$ [55"]. In this nonrandomized study, pulmonary capillary wedge pressure (PCWP) was reduced by $5.5 \mathrm{mmHg}$ at 30 days, and was 
accompanied by a reduction in NYHA class in all but one patient. The safety and efficacy of this device are currently being investigated in the Reduce Elevated Left Atrial Pressure in Patients with Heart Failure (REDUCE LAP-HF) Trial (NCT01913613). The V Wave device (V Wave Ltd, Hod HaSharon, Israel) applies the same approach to creating an interatrial shunt, but has the addition of a pericardial tissue valve to ensure one-way shunting. It recently underwent its first-in-man implantation in a patient with systolic dysfunction, and recruitment to a Phase 1 study is ongoing [56].

The CORolla device (Corassist Cardiovascular Ltd, Herzliya, Israel) is the first nonpharmacological approach to the problem of impaired ventricular relaxation. An elastic spring that is implanted in the left ventricular apex, the CORolla, absorbs energy during ventricular systole and then applies expansion forces to the septum and lateral wall during diastole, permitting increased ventricular filling. At this time, the device must be implanted surgically, but a transapical approach is under development.

\section{CONCLUSION}

HFpEF is characterized by a complex pathophysiology, encompassing multiple etiological mechanisms and exhibiting a diversity of clinical presentations. With a lack of proven therapies other than diuretics, HFpEF continues to have excessive morbidity and mortality. The failure to develop successful therapies for the management of HFpEF may be because of an overly broad disease definition and inadequate differentiation of disease subtypes. Developments in the understanding of pathophysiology, including the stages of disease, are fueling new therapies directed at improving both symptoms and survival (Table 3). Future trials may have greater success through targeting of specific subgroups or specific phases of disease development [57]. Recent successful physiological studies of heart rate reduction with ivabradine and neprilysin inhibition with LCZ696 have moved the field forward, and renewed excitement about new strategies for treating HFpEF. The results of the PARAGONHF study are eagerly awaited, as are the conclusion of multiple device trials that are currently in progress.

\section{Acknowledgments}

None.

\section{REFERENCES AND RECOMMENDED READING}

Papers of particular interest, published within the annual period of review, have been highlighted as:

- of special interest

- of outstanding interest

1. Redfield MM, Jacobsen SJ, Burnett JC Jr, et al. Burden of systolic and diastolic ventricular dysfunction in the community: appreciating the scope of the heart failure epidemic. JAMA. 2003; 289:194-202. [PubMed: 12517230]

2. Brouwers FP, de Boer RA, van der Harst P, et al. Incidence and epidemiology of new onset heart failure with preserved versus reduced ejection fraction in a community-based cohort: 11-year follow-up of PREVEND. Eur Heart J. 2013; 34:1424-1431. [PubMed: 23470495] 
3-. Wong CM, Hawkins NM, Petrie MC, et al. Heart failure in younger patients: the Meta-Analysis Global Group in Chronic Heart Failure (MAGGIC). Eur Heart J. 2014; 35:2714-2721. This meta-analysis of 24 prospective observational studies and seven randomized trials demonstrates improved survival in HFpEF compared with HFrEF, contradicting findings from earlier, smaller cohorts. [PubMed: 24944329]

4. Chung CS, Hutchinson KR, Methawasin M, et al. Shortening of the elastic tandem immunoglobulin segment of titin leads to diastolic dysfunction. Circulation. 2013; 128:19-28. A mouse model knockout altering titin isoform expression was associated with increased diastolic stiffness at the tissue and organ levels as well as with reduced exercise tolerance. [PubMed: 23709671]

5ロ. Hamdani N, Franssen C, Lourenco A, et al. Myocardial titin hypophosphorylation importantly contributes to heart failure with preserved ejection fraction in a rat metabolic risk model. Circ Heart Fail. 2013; 6:1239-1249. Cardiac titin hypophosphorylation was found to be associated with myocardial stiffness and HFpEF in an obese rat model with high metabolic risk. [PubMed: 24014826]

6. LeWinter MM, Meyer M. Mechanisms of diastolic dysfunction in heart failure with a preserved ejection fraction. Circ Heart Fail. 2013; 6:1112-1115. [PubMed: 24255055]

7. Lam CS, Roger VL, Rodeheffer RJ, et al. Cardiac structure and ventricular-vascular function in persons with heart failure and preserved ejection fraction from Olmsted County, Minnesota. Circulation. 2007; 115:1982-1990. [PubMed: 17404159]

8. Borlaug BA, Nishimura RA, Sorajja P, et al. Exercise hemodynamics enhance diagnosis of early heart failure with preserved ejection fraction. Circ Heart Fail. 2010; 3:588-595. [PubMed: 20543134]

9-m. Kraigher-Krainer E, Shah AM, Gupta DK, et al. Impaired systolic function by strain imaging in heart failure with preserved ejection fraction. J Am Coll Cardiol. 2014; 63:447-456. This substudy of the PARAMOUNT trial is an important contribution to the literature demonstrating the presence of systolic abnormalities in HFpEF. [PubMed: 24184245]

10-. Santos AB, Kraigher-Krainer E, Bello N, et al. Left ventricular dyssynchrony in patients with heart failure and preserved ejection fraction. Eur Heart J. 2014; 35:42-47. In this study, HFpEF was associated with greater dyssynchrony even in patients with LVEF higher than $55 \%$ and a narrow QRS interval. [PubMed: 24164863]

11. Santos AB, Kraigher-Krainer E, Gupta DK, et al. Impaired left atrial function in heart failure with preserved ejection fraction. Eur J Heart Fail. 2014; 16:1096-1103. This study uses strain imaging to identify abnormalities of left atrial function in HFpEF patients from the PARAMOUNT trial. [PubMed: 25138249]

12. Borlaug BA, Melenovsky V, Russell SD, et al. Impaired chronotropic and vasodilator reserves limit exercise capacity in patients with heart failure and a preserved ejection fraction. Circulation. 2006; 114:2138-2147. [PubMed: 17088459]

13. Borlaug BA. Mechanisms of exercise intolerance in heart failure with preserved ejection fraction. Circulation. 2014; 78:20-32.

14. Lund LH, Donal E, Oger E, et al. Association between cardiovascular vs. noncardiovascular comorbidities and outcomes in heart failure with preserved ejection fraction. Eur J Heart Fail. 2014; 16:992-1001. This observational prospective study demonstrated that HFpEF was associated with increased age, female sex, hypertension, atrial fibrillation and many noncardiovascular comorbidities. Mortality was predicted by noncardiovascular comorbidities, and was not predicted by cardiovascular comorbidities. [PubMed: 25046483]

15. Kitzman DW, Upadhya B. Heart failure with preserved ejection fraction: a heterogeneous disorder with multifactorial pathophysiology. J Am Coll Cardiol. 2014; 63:457-459. [PubMed: 24184240]

16". Zakeri R, Borlaug BA, McNulty SE, et al. Impact of atrial fibrillation on exercise capacity in heart failure with preserved ejection fraction: a RELAX trial ancillary study. Circ Heart Fail. 2014; 7:123-130. This substudy of the RELAX trial found that atrial fibrillation was associated with more advanced disease and reduced exercise capacity compared with sinus rhythm. [PubMed: 24162898]

17. Gori M, Senni M, Gupta DK, et al. Association between renal function and cardiovascular structure and function in heart failure with preserved ejection fraction. Eur Heart J. 2014; 
35:3442-3451. This study described a link between renal dysfunction and cardiac remodeling in HFpEF patients. [PubMed: 24980489]

18. Lindman BR, Davila-Roman VG, Mann DL, et al. Cardiovascular phenotype in HFpEF patients with or without diabetes: a RELAX trial ancillary study. J Am Coll Cardiol. 2014; 64:541-549. The negative impact of diabetes on ventricular hypertrophy, exercise capacity and hospitalizations is highlighted by this study. [PubMed: 25104521]

19m. Hwang SJ, Melenovsky V, Borlaug BA. Implications of coronary artery disease in heart failure with preserved ejection fraction. J Am Coll Cardiol. 2014; 62(25 Pt A):2817-2827. This observational study demonstrated that patients with HFpEF and coronary artery disease had increased mortality and greater deterioration in left ventricular ejection fraction compared with patients with HFpEF without coronary artery disease.

20. Akiyama E, Sugiyama S, Matsuzawa Y, et al. Incremental prognostic significance of peripheral endothelial dysfunction in patients with heart failure with normal left ventricular ejection fraction. J Am Coll Cardiol. 2012; 60:1778-1786. [PubMed: 23040568]

21-. Paulus WJ, Tschope C. A novel paradigm for heart failure with preserved ejection fraction: comorbidities drive myocardial dysfunction and remodeling through coronary microvascular endothelial inflammation. J Am Coll Cardiol. 2013; 62:263-271. In this review, Paulus and Tschope hypothesize that comorbidities cause symptoms in HFpEF by inducing a proinflammatory state that leads to coronary microvascular endothelial inflammation and subsequent diastolic dysfunction. [PubMed: 23684677]

22. Mohammed SF, Mirzoyev SA, Edwards WD, et al. Left ventricular amyloid deposition in patients with heart failure and preserved ejection fraction. JACC Heart Failure. 2014; 2:113-122. This autopsy study found evidence of senile amyloidosis in 5\% of HFpEF patients, and also identified mild amyloid deposition in another $12 \%$ of patients. Amyloid deposition was associated with fibrosis even after adjustment for age. [PubMed: 24720917]

23m. Shah AM, Shah SJ, Anand IS, et al. Cardiac structure and function in heart failure with preserved ejection fraction: baseline findings from the echocardiographic study of the Treatment of Preserved Cardiac Function Heart Failure with an Aldosterone Antagonist trial. Circ Heart Fail. 2014; 7:104-115. This study from the echocardiography core laboratory of the TOPCAT trial identifies the prevalence of different forms of ventricular remodeling in HFpEF. [PubMed: 24249049]

24. Gori M, Lam CS, Gupta DK, et al. Sex-specific cardiovascular structure and function in heart failure with preserved ejection fraction. Eur J Heart Fail. 2014; 16:535-542. [PubMed: 24574260]

25. Melenovsky V, Hwang SJ, Lin G, et al. Right heart dysfunction in heart failure with preserved ejection fraction. Eur Heart J. 2014; 35:3452-3462. [PubMed: 24875795]

26-. Mohammed SF, Hussain I, Abou Ezzeddine OF, et al. Right ventricular function in heart failure with preserved ejection fraction: a community based study. Circulation. 2014; 130:2310-2320. This prospective community-based study of 562 patients demonstrates that right ventricular dysfunction in HFpEF is associated with increased mortality and heart failure hospitalizations. [PubMed: 25391518]

27. Bonderman D, Pretsch I, Steringer-Mascherbauer R, et al. Acute hemodynamic effects of riociguat in patients with pulmonary hypertension associated with diastolic heart failure (DILATE-1): a randomized double-blind, placebo-controlled, single-dose study. Chest. 2014; 146:1274-1285. In a small ( $n=21)$ study, riociguat did not improve mean pulmonary artery pressure but did improve stroke volume and reduce right ventricular size as compared with placebo. [PubMed: 24991733]

28. Zile MR, Bourge RC, Redfield MM, et al. Randomized double-blind, placebo-controlled study of sitaxsentan to improve impaired exercise tolerance in patients with heart failure and a preserved ejection fraction. JACC Heart Fail. 2014; 2:123-130. In this placebo-controlled trial of 192 patients with $\mathrm{HFpEF}$, the group treated with the selective endothelin type A receptor antagonist sitaxsentan had increased exercise tolerance compared with the placebo group, but no changes in diastolic function or symptoms. [PubMed: 24720918]

29m. Adamson PB, Abraham WT, Bourge RC, et al. Wireless pulmonary artery pressure monitoring guides management to reduce decompensation in heart failure with preserved ejection fraction. Circ Heart Fail. 2014; 7:935-944. In this substudy of the CHAMPION trial, implantable 
pulmonary artery monitoring showed promise for the management of outpatients with HFpEF in terms of preventing hospitalizations. [PubMed: 25286913]

30-. Kitzman DW, Brubaker PH, Herrington DM, et al. Effect of endurance exercise training on endothelial function and arterial stiffness in older patients with heart failure and preserved ejection fraction: a randomized, controlled, single-blind trial. J Am Coll Cardiol. 2013; 62:584592. In 63 patients with $\mathrm{HFpEF}$, randomization to exercise training for 16 weeks increased peak $\mathrm{VO}_{2}$ and quality of life, but did not affect measures of endothelial function. [PubMed: 23665370]

31. Pandey A, Parashar A, Kumbhani D, et al. Exercise training in patients with heart failure and preserved ejection fraction: a meta-analysis of randomized control trials. Circ Heart Fail. 2015; 8:33-40. This meta-analysis reported that exercise training for patients with HFpEF improves quality of life and exercise capacity. [PubMed: 25399909]

32. Yusuf S, Pfeffer MA, Swedberg K, et al. Effects of candesartan in patients with chronic heart failure and preserved left-ventricular ejection fraction: the CHARM-Preserved Trial. Lancet. 2003; 362:777-781. [PubMed: 13678871]

33. Cleland JG, Tendera M, Adamus J, et al. The Perindopril in Elderly People with Chronic Heart Failure (PEP-CHF) study. Eur Heart J. 2006; 27:2338-2345. [PubMed: 16963472]

34. Massie BM, Carson PE, McMurray JJ, et al. Irbesartan in patients with heart failure and preserved ejection fraction. N Engl J Med. 2008; 359:2456-2467. [PubMed: 19001508]

35. Edelmann F, Wachter R, Kraigher-Krainer E, et al. Effect of spironolactone on diastolic function and exercise capacity in patients with heart failure with preserved ejection fraction. JAMA. 2013; 309:781-791. [PubMed: 23443441]

36-m. Pitt B, Pfeffer MA, Assmann SF, et al. Spironolactone for heart failure with preserved ejection fraction. N Engl J Med. 2014; 370:1383-1392. The TOPCAT trial is a large randomized study that was powered to investigate the effect of aldosterone antagonism on clinical outcomes in HFpEF. At a mean follow-up of 38 months, there was no significant difference in the primary endpoint of death from cardiovascular causes, aborted cardiac arrest or hospitalization for heart failure. [PubMed: 24716680]

37. Pfeffer MA, Claggett B, Assmann SF, et al. Regional variation in patients and outcomes in the Treatment of Preserved Cardiac Function Heart Failure with an Aldosterone Antagonist Trial. Circulation. 2015; 131:34-42. This substudy of the TOPCAT trial identifies different event rates and treatment effects based on the region where patients were enrolled. This suggests the conclusion that the failure of TOPCAT may have been related to the enrollment of some patients who were not the target population. [PubMed: 25406305]

38. Kristensen SL, Kober L, Jhund PS, et al. International geographic variation in event rates in trials of heart failure with preserved and reduced ejection fraction. Circulation. 2015; 131:43-53. This analysis of patients from five major international heart failure studies showed marked variation in event rates for HFpEF patients across different geographic regions, suggesting the need for a stricter definition of HFpEF in trial inclusion criteria. [PubMed: 25406306]

39-. Shah AM, Claggett B, Sweitzer NK, et al. Cardiac structure and function and prognosis in heart failure with a preserved ejection fraction: findings from the echocardiographic study of the Treating Preserved Cardiac Function Heart Failure with an Aldosterone Antagonist (TOPCAT) Trial. Circ Heart Fail. 2014; 7:740-751. This substudy of the TOPCAT trial makes an important contribution to the understanding of how phenotypic presentations of HFpEF are associated with prognosis. In this study, left ventricular hypertrophy, higher left ventricular filling pressure and higher pulmonary artery pressure were associated with an increased incidence of the primary endpoint. [PubMed: 25122186]

40. Nagayama T, Hsu S, Zhang M, et al. Sildenafil stops progressive chamber, cellular, and molecular remodeling and improves calcium handling and function in hearts with preexisting advanced hypertrophy caused by pressure overload. J Am Coll Cardiol. 2009; 53:207-215. [PubMed: 19130990]

41. Guazzi M, Vicenzi M, Arena R, Guazzi MD. Pulmonary hypertension in heart failure with preserved ejection fraction: a target of phosphodiesterase-5 inhibition in a 1 year study. Circulation. 2011; 124:164-174. [PubMed: 21709061]

42-. Redfield MM, Chen HH, Borlaug BA, et al. Effect of phosphodiesterase-5 inhibition on exercise capacity and clinical status in heart failure with preserved ejection fraction: a randomized clinical 
trial. JAMA. 2013; 309:1268-1277. This was a multicenter randomized controlled trial assessing the effect of sildenafil on exercise capacity and clinical status in patients with HFpEF. The results of this trial showed no significant difference between sildenafil and placebo in exercise capacity, clinical status or secondary endpoints. [PubMed: 23478662]

43-. Kosmala W, Holland DJ, Rojek A, et al. Effect of If-channel inhibition on hemodynamic status and exercise tolerance in heart failure with preserved ejection fraction: a randomized trial. $\mathrm{J}$ Am Coll Cardiol. 2013; 62:1330-1338. This small clinical study of ivabradine showed significant improvements in exercise capacity, peak oxygen uptake and diastolic function as measured by echocardiography. Ivabradine may be a promising therapy for patients with symptoms that only occur during exercise. [PubMed: 23916925]

44. Bohm M, Perez AC, Jhund PS, et al. Relationship between heart rate and mortality and morbidity in the Irbesartan patients with heart failure and preserved systolic function trial (I-Preserve). Eur J Heart Fail. 2014; 16:778-787. Analysis of the I-Preserve trial revealed that for patients with HFpEF in normal sinus rhythm, increased heart rates were associated with an increased composite risk of cardiovascular death or heart failure hospitalization. [PubMed: 24864045]

45. Solomon SD, Zile M, Pieske B, et al. The angiotensin receptor neprilysin inhibitor LCZ696 in heart failure with preserved ejection fraction: a phase 2 double blind randomized controlled trial. Lancet. 2012; 380:1387-1395. [PubMed: 22932717]

46. Pieske B, Butler J, Filippatos G, et al. Rationale and design of the SOluble guanylate Cyclase stimulatoR in heArT failurE Studies (SOCRATES). Eur J Heart Fail. 2014; 16:1026-1038. [PubMed: 25056511]

47. Reil JC, Hohl M, Reil GH, et al. Heart rate reduction by If-inhibition improves vascular stiffness and left ventricular systolic and diastolic function in a mouse model of heart failure with preserved ejection fraction. Eur Heart J. 2013; 34:2839-2849. Mouse model data indicates improvements in vascular stiffness, ventricular elastance and diastolic function with ivabradine, an $\mathrm{I}_{\mathrm{f}}$-channel inhibitor that works directly on the sinus node to reduce chronotropy without negative inotropy. [PubMed: 22833515]

48. Jhund PS, Claggett B, Packer M, et al. Independence of the blood pressure lowering effect and efficacy of the angiotensin receptor neprilysin inhibitor, LCZ696, in patients with heart failure with preserved ejection fraction: an analysis of the PARAMOUNT trial. Eur J Heart Fail. 2014; 16:671-677. [PubMed: 24692284]

49. Bonderman D, Ghio S, Felix SB, et al. Riociguat for patients with pulmonary hypertension caused by systolic left ventricular dysfunction. Circulation. 2013; 128:502-511. [PubMed: 23775260]

50. Borlaug BA, Melenovsky V, Russell SD, et al. Impaired chronotropic and vasodilator reserves limit exercise capacity in patients with heart failure and a preserved ejection fraction. Circulation. 2006; 114:2138-2147. [PubMed: 17088459]

51. Kass DA, Kitzman DW, Alvarez GE. The restoration of chronotropic competence in heart failure patients with normal ejection fraction (RESET) study: rationale and design. J Card Fail. 2010; 16:17-24. [PubMed: 20123314]

52. Laurent G, Eicher JC, Mathe A, et al. Permanent left atrial pacing therapy may improve symptoms in heart failure patients with preserved ejection fraction and atrial dyssynchrony: a pilot study prior to a national clinical research programme. Eur J Heart Fail. 2013; 15:85-93. [PubMed: 23018991]

53. Topilsky Y, Pereira NL, Shah DK, et al. Left ventricular assist device therapy in patients with restrictive and hypertrophic cardiomyopathy. Circulation. 2011; 4:266-275.

54. Kaye D, Shah SJ, Borlaug BA, et al. Effects of an interatrial shunt on rest and exercise hemodynamics: results of a computer simulation in heart failure. J Card Fail. 2014; 20:212-221. [PubMed: 24487087]

55-. Sondergaard L, Reddy V, Kaye D, et al. Transcatheter treatment of heart failure with preserved or mildly reduced ejection fraction using a novel interatrial implant to lower left atrial pressure. Eur J Heart Fail. 2014; 16:796-801. This is the first report of using a device to create an interatrial shunt for decompression of the left atrium, a promising new therapy for HFpEF. [PubMed: 24961390]

56. Amat-Santos IJ, Bergeron S, Bernier M, et al. Left atrial decompression through unidirectional left-to-right interatrial shunt for the treatment of left heart failure: first-in-man experience with the V-Wave device. Euro Intervention. 2015; 10:1127-1131. [PubMed: 24832489] 
57. Shah SJ. Matchmaking for the optimization of clinical trial of heart failure with preserved ejection fraction: no laughing matter. J Am Coll Cardiol. 2013; 62:1339-1342. [PubMed: 23916923] 


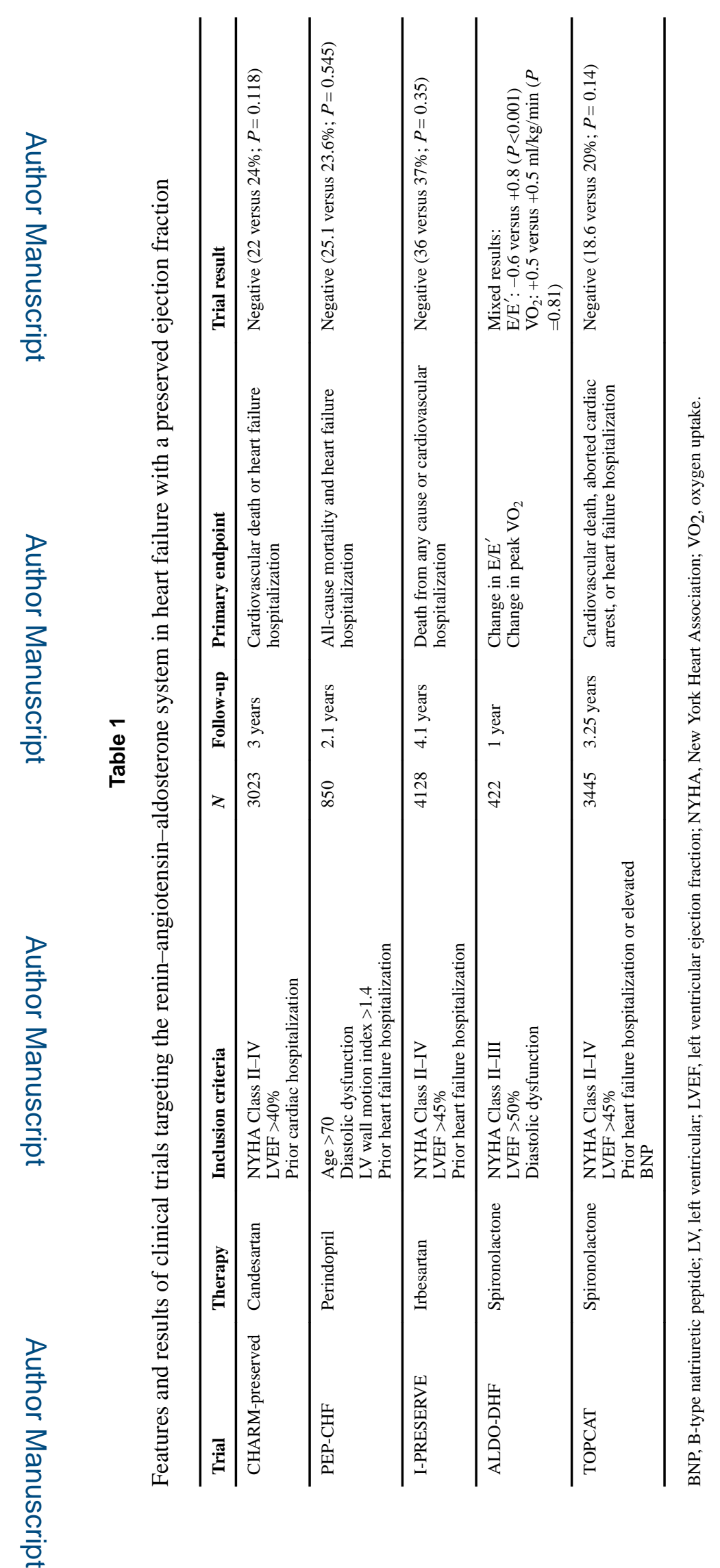

Curr Opin Cardiol. Author manuscript; available in PMC 2017 April 14 


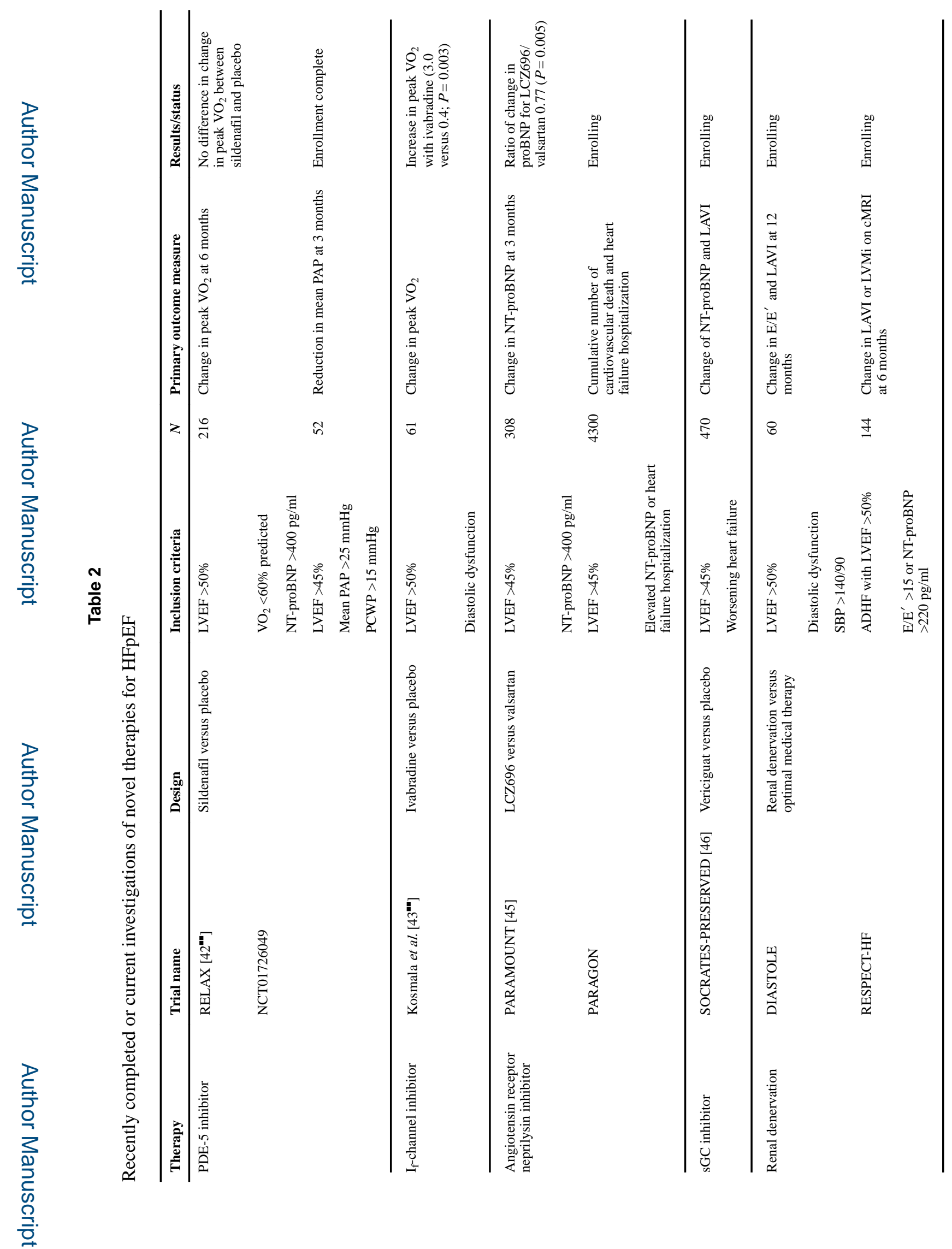

Curr Opin Cardiol. Author manuscript; available in PMC 2017 April 14. 

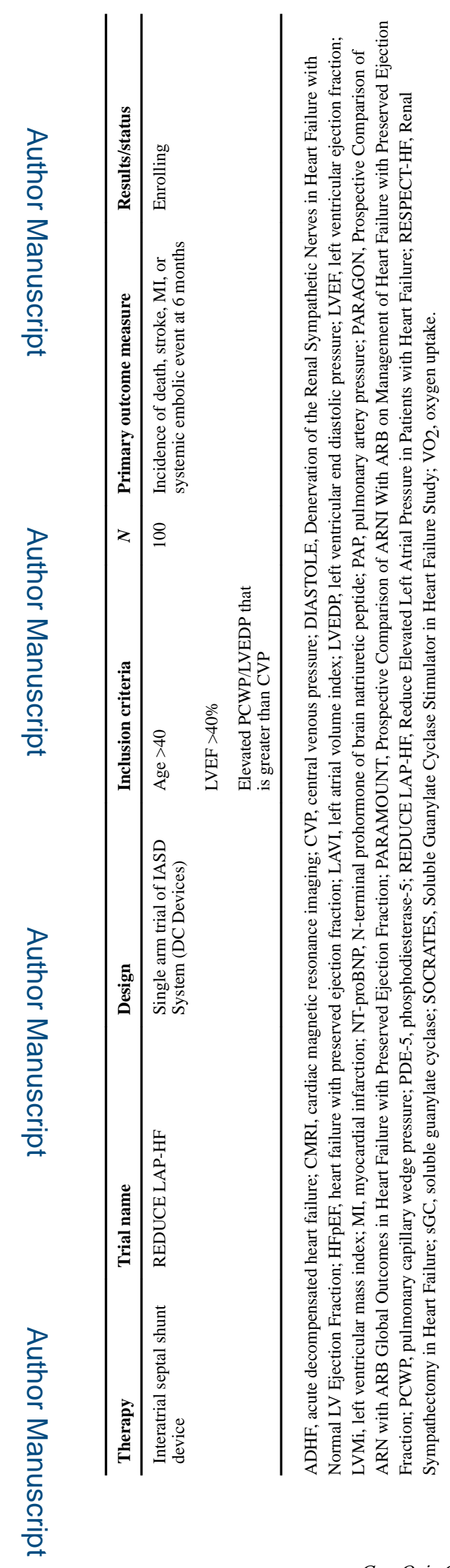

Curr Opin Cardiol. Author manuscript; available in PMC 2017 April 14. 
Table 3

Examples of targeted therapies based on specific pathophysiologic mechanisms in HFpEF

\begin{tabular}{ll}
\hline Targeted etiological mechanisms & Potential therapies \\
\hline Exertional dyspnea (exercise-induced diastolic dysfunction) & Ivabradine exercise training, interatrial shunt devices \\
\hline Volume overload & Pulmonary artery pressure monitor, angiotensin receptor neprilysin inhibitor \\
\hline Pulmonary hypertension and right ventricular dysfunction & Phosphodiesterase-5 inhibitors, soluble guanylate cyclase stimulators \\
\hline Impaired ventricular relaxation & Intraventricular spring with diastolic expansion \\
\hline Chronotropic incompetence & Rate-adaptive atrial pacing \\
\hline Atrial dyssynchrony & Left atrial pacing \\
\hline
\end{tabular}

$\mathrm{HFpEF}$, heart failure with preserved ejection fraction. 